\title{
PERCEPCIÓN DE CALIDAD DE VIDA LABORAL EN UNA EMPRESA FARMACEUTICA
}

\section{PERCEPTION OF QUALITY OF WORKING LIFE IN A PHARMACEUTICAL COMPANY}

\section{ASHLEY ALBARRASIN-VELÁSQUEZ ${ }^{1}$ KARINA SILVA-JARAMILLO ${ }^{2}$ VERÓNICA GARCÍA-OQUENDO ${ }^{3}$}

Recibido: 15 de mayo de 2017 Aceptado: 29 de agosto de 2017

\footnotetext{
1 Deloitte Ecuador, Quito, Ecuador (ashleym_a93@hotmail.com).

${ }^{2}$ Pontificia Universidad del Ecuador, Facultad de Psicología, Quito, Ecuador (ksilvao66@puce.edu.ec).

3 Pontificia Universidad del Ecuador, Facultad de Psicología, Quito, Ecuador (mvgarcia@puce.edu.ec).
} 



\section{PERCEPCIÓN DE CALIDAD DE VIDA LABORAL EN UNA EMPRESA FARMACEUTICA}

\section{PERCEPTION OF QUALITY OF WORKING LIFE IN A PHARMACEUTICAL COMPANY}

Ashley Albarrasin-Velásquez, Karina Silva-Jaramillo, Verónica García-Oquendo

PALABRAS CLAVE: Calidad de vida laboral, instrumentos de medición, equilibrio trabajo-familia, dedicación al trabajo

KEY WORDS: Quality of working life, measuring instruments, work-family balance, dedication to work

\section{RESUMEN}

En las últimas décadas la Calidad de Vida Laboral (CVL) ha generado interés en el ámbito de la psicología organizacional debido al cuidado del bienestar del capital humano: motor principal de cualquier organización. El objetivo del presente estudio es determinar el nivel de CVL en colaboradores del área de ventas de una farmacéutica en la ciudad de Quito a través de la aplicación del cuestionario CVT-GOHISALO a una muestra $(n=79)$. El perfil de CVL de estos colaboradores evidencia promedios de satisfacción en la mayoría de las dimensiones estudiadas. Sin embargo, presenta un bajo puntaje en la dimensión de administración del tiempo libre, resultado interesante considerando las características inherentes a su trabajo. 


\section{ABSTRACT}

In the last decades, the Quality of Work Life (QWL) has generated interest in the field of organizational psychology due to the welfare of human capital: the main engine of any organization. The objective of the present study is to determine the QWL level in collaborators of the sales area of a pharmaceutical company in the city of Quito through the application of the CVT-GOHISALO questionnaire to a sample $(n=79)$. The QWL profile of these collaborators evidences averages of satisfaction in most of the studied dimensions, however presents a low score in the administration dimension of free time, an interesting result considering the inherent characteristics of their work.

\section{INTRODUCCIÓN}

En las últimas décadas, la Calidad de Vida Laboral (CVL) ha sido uno de los conceptos que más interés ha generado en las empresas que buscan una manera diferente de sobresalir ante su competencia e imponerse en el mercado, interesándose y cuidando su capital humano. Se ha considerado un campo emergente y desafiante para el campo de la psicología organizacional. La CVL se define como "las condiciones y ambientes de trabajo favorables que protegen y promueven la satisfacción de los empleados mediante recompensas, seguridad laboral y oportunidades de desarrollo personal" (Segurado \& Agulló, 2002, pág. 830).

En el entorno empresarial, la CVL está relacionada con las compensaciones intangibles; beneficios, plan de carrera y sucesión, diversidad laboral, conciliación entre el trabajo-familia, flexibilidad de horarios; en general con la salud y el bienestar (Chiang Vega \& Krausse Martínez, 2009). También se establecen políticas, programas y prácticas de recursos humanos como estrategia para la retención de colaboradores con alto potencial, además de cumplir con requisitos mínimos de responsabilidad social empresarial.

Katzell, Yankelovich, Fein, Ornati y Nash (1975) citados en (Martínez Gamarra \& Ros Mar, 2010) exponen que una alta CVL está relacionada con los sentimientos positivos del colaborador hacia su trabajo; es decir, le permite percibir la existencia de una armonía entre su vida privada y su actividad laboral. Se pueden destacar dos aspectos de la CVL: 1) sensación positiva hacia el trabajo que puede ser catalogada 
como clima laboral, satisfacción laboral, motivación, compromiso; 2) el significado personal de trabajo que puede ser pertenencia social, como un deber u obligación para encajar en los sistemas sociales (Gestoso, 2000). Dependiendo de cuál sea la concepción en los individuos, se articula las estrategias de la conciliación entre la vida laboral y la familia.

Martínez San Esteban y Calvo Lluch (2014) establecen que la CVL percibida tiene una relación positiva con los trabajadores que realizan alguna actividad física regular, en comparación con aquellos que no la realizan. En esta misma línea, Moreno, Cueva y Gonzalez (2009, pág. 56) encontraron "relaciones positivas significativas entre los indicadores de calidad de vida y la percepción de salud física y psicológica y satisfacción". Asimismo encuentran, relaciones significativas negativas con el estrés, el agotamiento y la intención de renunciar. Estos mismos autores mencionan la investigación de Golden (2005) que estudió la relación entre el tiempo extraordinario, la percepción de bienestar físico y mental de los colaboradores y una relación significativa entre el trabajo realizado en jornadas extraordinarias y vida familiar.

Existen diferentes marcos internacionales y nacionales que promueven y velan por el bienestar de los trabajadores. La Organización Internacional del trabajo (OIT) que defiende y promueve el Tra- bajo Decente; es decir, que las personas durante su vida laboral tengan oportunidad de un ingreso justo, "seguridad en el lugar de trabajo y protección social para las familias, mejores perspectivas de desarrollo personal e integración social, libertad para que los individuos expresen sus opiniones, se organicen y participen en las decisiones que afectan sus vidas, y la igualdad de oportunidades y trato para todos: mujeres y hombres" (Organización Internacional del Trabajo, 2017).

En el Ecuador, el noveno objetivo del Plan Nacional del Buen Vivir (20132017) contempla: "garantizar el trabajo digno en todas sus formas, cuya meta es brindar condiciones para que los colaboradores tengan medios para ejecutar sus actividades y sobre todo se reconoce la supremacía del trabajo humano sobre el capital" (Secretaria Nacional de Planificación y Desarrollo, 2017).

Los instrumentos de medición más utilizados en América Latina de acuerdo a la literatura revisada son: calidad de vida profesional (CVP-35) con tres dimensiones; perfil de calidad de vida laboral (PCVL) con ocho dimensiones; CVT-GOHISALO con siete dimensiones; cuestionario de bienestar laboral general (Qblg) con ocho dimensiones; Q-Laboral 56 con 10 dimensiones y Professional quality of life (ProQOL) con tres dimensiones.

Considerando que la CVL es fenómeno social, debe continuar siendo 
investigado desde diversas áreas del conocimiento y contextualizado en una sociedad pos-moderna como la nuestra. El objetivo del presente estudio es determinar el nivel de CVL en colaboradores del área de ventas (visitadores médicos) de una farmacéutica en Quito a través de la aplicación del cuestionario CVT-GOHISALO a una muestra $(n=79)$.

\section{MARCO CONCEPTUAL}

Los orígenes de la CVL están relacionados con los trabajos realizados en el Instituto Tavistock de relaciones humanas en Londres, en donde se da un primer enfoque a este concepto desde una apreciación sociopsicológica (Gómez, 2010), a partir de la cual surge el desarrollo socio técnico del mismo, al ser aplicado en el campo organizacional. Para Emery (1959) citado en Manrique (2013), un sistema socio técnico trata los aspectos sociales de las personas, la colectividad y los aspectos técnicos de la organización: su estructura y procesos. Aparecen propuestas organizacionales desarrolladas con base en la apreciación sociopsicológica y son recopiladas por el movimiento de la Democracia Industrial en los países escandinavos. Este movimiento tenía por objetivo buscar el desarrollo de formas alternativas de organización del trabajo, en donde participen los trabajadores de distintos niveles, sustituyendo las formas altamente jerárquicas por otras más flexibles (Peiró, 1996).
Paralelamente a este movimiento, en los Estado Unidos surge el movimiento del Desarrollo Organizacional que busca mejorar la eficiencia y eficacia en la organización sin descuidar el bienestar de sus trabajadores (Peiró, 1996). Es así que, a partir de los planteamientos del enfoque socio técnico sobre el rediseño del trabajo y el movimiento del Desarrollo Organizacional, se sentaron las bases para el desarrollo del movimiento CVL. EI cual parte de la necesidad de humanizar el entorno del trabajo prestando especial atención al desarrollo humano y a la mejora de su calidad de vida (Segurado \& Agulló, 2002). A partir de este momento, el tema de la CVL se popularizó tanto en los Estados Unidos como en Europa en donde la situación laboral contaba con altos niveles de precariedad.

El termino CVL fue utilizado por primera vez por Louis Davis en 1970, para describir la preocupación que toda organización debía tener por el bienestar y la salud de todos sus empleados con el objetivo de obtener y mantener un alto 
desempeño (Gómez, 2010). Desde la aparición de este concepto en los años 70, varios han sido los estudios y publicaciones Ilevados a cabo con la finalidad de facilitar la definición del término. Las conceptualizaciones más clásicas corresponden a los inicios del movimiento de $C V L$, que considera un enfoque amplio y genérico basado en la valoración del individuo con relación a su medio de trabajo; es decir, desde una perspectiva subjetiva (Segurado \& Agulló, 2002).

Más tarde, la CVL empieza a tratarse desde el punto de vista de la empresa, una perspectiva mucho más objetiva, haciendo alusión a los distintos procesos y dinámicas organizacionales que afectan al trabajador (Segurado \& Agulló, 2002).

Hoy en día, el concepto de CVL se sustenta en dos aspectos muy importantes; los de percepción y satisfacción, en los cuales se incluyen tanto aspectos físicos y ambientales, como aspectos psicológicos de los colaboradores en la organización. Estos aspectos son comprendidos según los procesos cognitivos individuales basados en los impulsos que reciban (percepción) para hacerlos sentir bien o complacidos según se haya cubierto una necesidad (satisfacción) a través del trabajo (González, Hidalgo, León, Contreras, Alderete, Hidalgo y Barrera, 2015).

Como indica Chiavenato (2007), la CVL no solo implica los aspectos in- trínsecos al puesto, sino también todos los aspectos extrínsecos y contextuales. Es por esto que para fines de esta investigación se tomó como referencia su concepto de CVL, el cual plantea el "grado en que los miembros de la organización pueden satisfacer sus necesidades personales con su actividad en la organización" (pág. 351).

En la actualidad, no existe una sola definición de CVL debido a que esta se relaciona con todos los aspectos del trabajo que influyan en la satisfacción, la motivación, el compromiso laboral y el rendimiento laboral. Por estos motivos la CVL se ha convertido en un concepto multidimensional, provocando así numerosos estudios y publicaciones con distintos enfoques en cuanto a dimensiones que componen la CVL. Estas dimensiones se agrupan en dos grandes bloques: los aspectos de la CVL que tienen relación con el entorno en que se realiza el trabajo o condiciones objetivas y las concernientes con la experiencia psicológica de los trabajadores o condiciones subjetivas (Casas, Repullo, \& Cañas, 2002).

Casas et al. (2002) definen las dimensiones conceptuales más representativas y relevantes del constructo de $C V L$ en el sector sanitario: condiciones objetivas (medios físicos, tecnológicos, contractuales, productivos y profesionales) y las condiciones subjetivas (esfera 
privada y mundo laboral, individuo y actividad profesional, individuo y grupo laboral, individuo e institución).

Dentro de las condiciones objetivas, el medio físico constituye la parte concreta del medio ambiente productivo. Casas et al. (2002) consideran las jornadas laborales y la carga de trabajo como factores influyentes en la calidad de vida laboral. El trabajar en un horario no establecido o por turnos, así como trabajar en un horario nocturno supone una gran dificultad en el mantenimiento de una vida familiar y social normales. Además, es frecuente que los trabajadores se quejen de problemas en su salud como problemas de sueño, fatiga o alteraciones en su ciclo circadiano. La falta de tareas durante la jornada laboral, la asignación de tareas rutinarias o aburridas o la sobrecarga laboral son condiciones del medio ambiente que producen insatisfacción y, por lo tanto, afecta la percepción de la CVL.

Por otro lado, la conciliación laboral puede ser considerada como una condición objetiva, tecnología, contractual y productiva. Se refiere a las medidas de flexibilidad temporal y espacial que le permiten al colaborador combinar mejor las obligaciones laborales con las responsabilidades familiares o expectativas de ocio. Actualmente, las organizaciones han desarrollado diversas medidas como políticas de conciliación entre las que se pueden mencionar: horarios variables o flexibles, reducción de horas laborales, permisos, teletrabajos, entre otros. Eaton, (2003) citado por Pasamar \& Valle, 2011.

En lo profesional se puede hacer referencia a la perspectiva de género que también es utilizada como estrategia de bienestar para la conciliación laboral, es reconocido en la sociedad el papel fundamental de las mujeres en la actividad económica, en la vida familiar y su repercusión en la sociedad (González et al., 2015). La familia es considerada como un elemento importante en la vida de los sujetos puesto que contribuye significativamente al bienestar, es una institución modificable y perenne, según Guerrero (2003); Ardila Goméz, (2009) citado por Jiménez, Figueroa, Mendiburro, Subiabre \& Olmedo Fuentes (2011) .

Dentro de las condiciones subjetivas se encuentra la esfera de vida privada y el mundo laboral, misma que hace referencia al entorno empresarial. Entorno que está relacionado con la planificación del tiempo libre, el equilibrio entre trabajo y vida familiar y la dedicación al trabajo. Como indican McMillan, Lane y Atchley (2011), citados en Valadez (2012), la vida laboral y la vida familiar son dimensiones que se influyen mutuamente. Las experiencias y las decisiones que ocurren en un ámbito pueden intervenir en los resultados de otro, por lo que equilibrar las responsabilidades familia- 
res y laborales constituye un factor que necesariamente debe ser considerado. El balance vida-trabajo se logra cumpliendo con todos los roles en el ámbito familiar sin descuidar las responsabilidades laborales; ya que si no se lograse encontrar una conciliación entre estas dos dimensiones, la tensión que se genere por este conflicto puede manifestarse en un nivel de estrés que impacte de manera negativa a la salud del colaborador, su estado de ánimo, eficiencia, relaciones inter-personales, etc. (Salgado, 2011); (Valadez, 2012).

Asimismo, el individuo y la actividad profesional también forman parte de las condiciones subjetivas. Las organizaciones deben alcanzar sus objetivos para subsistir; en esta dinámica se pueden generar largas jornadas laborales, prácticas que en el mediano plazo traen consecuencias negativas tanto para el desempeño organizacional como para el colaborador, entre las que se podría mencionar insatisfacción laboral, falta de compromiso, ausentismo y rotación. Por otra parte, el trabajo conlleva satisfacciones con la adecuada estructura organizacional y una supervisión presente brinda al colaborador elementos que conciban en corto plazo satisfacción laboral. Además, si las empresas ofrecen planes de desarrollo y directrices claras y coherentes podría disminuir el hecho de las jornadas extensas.
Por otro lado, los individuos consideran el tiempo con un recurso valioso y escaso, la planificación del tiempo libre ha sido afectada de cierta manera por las exigencias laborales y dedicación al trabajo. Se debe contextualizar que en nuestra sociedad pos-moderna existe una gran tendencia al consumismo, por tal motivo los trabajadores tienen valores, desean éxitos y metas capitalistas orientándose a incrementar sus niveles de ingreso económico dedicando horas y esfuerzo a su trabajo para conseguir un cierto estilo de vida (Brett \& Stroh, 2003) en (Guerrero \& Puerto Barios, 2007).

Una de las mayores preocupaciones en el ámbito familiar es la crianza de los hijos, en este aspecto entra el concepto de "corresponsabilidad parental" que según Paterna y Martínez (2009) citado por Ortega, Rodríguez y Jiménez (2013) se define "tres componentes principales: compromiso, es decir, tiempo en interacción cara a cara con el niño; accesibilidad: estar física y psicológicamente presente para atender las necesidades del niño; y responsabilidad: asumir las tareas de cuidado y bienestar día a día" (pág. 56).

Para Greenhaus, Collins \& Shaw (2003) se debe comprender la armonía entre las actividades laborales, los roles que se representan y la vida familiar con sus exigencias como la distribución del tiempo libre para actividades que no es- 
tén relacionadas con el trabajo, el cuidado de la familia y su función parental y otras actividades de ocio. Asimismo, para Kirchmeyer (2000), citado en Figueroa (2008), el equilibrio es una percepción de satisfacción en las otras esferas de la vida que demandan tiempo, recursos personales y compromiso para distribuirse adecuadamente en todos los ámbitos. En definitiva, es el funcionamiento y operalización de los roles que pueden generar conflicto considerando los tres componentes del equilibrio: tiempo, niveles de compromiso y satisfacción en los dominios de trabajo y vida familiar.

De igual manera, la satisfacción en el trabajo tiene igual o mayor peso en el bienestar global, la conciliación trabajo-familia es una base sustancial para el ajuste psicológico del individuo porque lo que puede causar conflictos potenciales, ambas esferas son dependientes y confusas en sus límites para Greenhous et al. (2003); Shiffley, (2003); Jiménez y Moyano (2008); Pasaruraman, Greenhous y Rabinowitz, (1989) citado en (Jiménez et al., 2011).

Presiones laborales como jornadas de trabajo prolongadas, características en sí de la tarea como viajes continuos, horarios de fines de semana, complicaciones cotidianas del desempeño de su labor como la constante movilización en grandes metrópolis pueden acarear potenciales conflictos en la esfera familiar. (Torres Martos \& Onsalo, 2012). El conflicto surge cuando el individuo asume varios roles entre su trabajo y familia, los cuales pueden ser antagónicos e incompatibles generando altos niveles de estrés e insatisfacción laboral en el ámbito organizacional mientras que en la esfera familiar se evidencia detrimento en la función parental; además, reflejan tensión, ansiedad, depresión y problemas psicosomáticos. Frone, Russell \& Cooper, (1997); McFarland, (2004); Cifre y Salanova, (2004) referidos por (Jiménez et al., 2011).

Es relevante mencionar los modelos desarrollados para encontrar las causas y relaciones en la dinámica trabajo-familia; por ejemplo, el Modelo Spillover y el Modelo Compensatorio. El primero postula los límites físicos, temporales y emocionales-conductuales que interaccionan entre los dominios. El segundo está relacionado con el esfuerzo que realiza el individuo para compensar las debilidades de un ámbito en relación al otro; sin embargo, desde la perspectiva de la familia se puede encontrar individuos insatisfechos con sus relaciones interfamiliares, motivo por el cual dedican más tiempo a su trabajo. En investigaciones recientes se menciona el planteamiento "Border Theory" que evidencia como el colaborador negocia constantemente el plano familiar con el trabajo y la influencia recíproca en cada 
dominio. Clarck (2000); Edwards \& Rothbard (2000) en (Figueroa, 2008).

Con todos estos antecedentes, es innegable discutir la complejidad multidimensional de la CVL y de las dificultades que supone la medición de la misma. Es importante considerar que cuando se plantee una medición de $C V L$, el instrumento que vaya a uti- lizarse debe buscar identificar aquellas dimensiones y componentes de las mismas que definan problemas y oportunidades de mejora y desarrollo para la empresa. Finalmente, al escoger un instrumento para medir la CVL, se deben considerar las variables organizaciones con las cuales se puede trabajar e intervenir.

\section{MATERIALES Y MÉTODOS}

\section{Participantes}

Para esta investigación se trabajó en función de una muestra por conveniencia $(n=79)$ compuesta por colaboradores de la fuerza de ventas, entre los que se encuentran visitadores médicos, representantes institucionales, gerente de distrito, KIM (Key International Manager) y KAM (Key account manager). Los criterios de inclusión fueron: 1) que trabajen en Quito y 2) antigüedad mayor a tres meses. Esta muestra estuvo compuesta en un $51 \%$ por hombres y un $49 \%$ de mujeres, quienes en su mayoría se encuentran casados, con edades comprendidas entre los 41 y 50 años y con 2 hijos en promedio.

\section{Metodología}

El diseño de esta investigación fue de carácter descriptivo y se midieron 7 dimensiones de la CVL: soporte institu- cional para el trabajo (SIT), seguridad en el trabajo (ST), integración al puesto de trabajo (IPT), satisfacción por el trabajo (SAT), bienestar logrado a través del trabajo(BLT), desarrollo personal (DP) y administración del tiempo libre (ALT).

El instrumento aplicado fue el cuestionario CVT-GOHISALO, debido a que cuenta con una validación en poblaciones similares (alfa de Cronbach 0.64 a 0.93 en mexicana y peruana) a la ecuatoriana y, además, es uno de los más completos en cuanto a medición de CVL. Se realizó una validación lingüística del mismo con el objetivo de que las preguntas mantenidas en el cuestionario estuvieran completamente claras y así obtener resultados más confiables. Se aplicó un pre-test con una muestra a conveniencia de dos visitadores médicos por cada línea de negocio representativa: movilidad, gastropediatria y genfar $(n=.6)$. Se 
reformularon 10 preguntas con la validación lingüística.

La aplicación del instrumento fue por medio de una plataforma interna de la empresa, en forma de encuesta facilitando así la recolección de datos. Se utilizó la escala de Likert para el análisis de los resultados obtenidos para cada dimensión y sub-dimensión evaluada, en donde 0 es el puntaje mínimo y 4 el puntaje máximo. Posteriormente se calculó un promedio para cada dimensión, sumando los datos obtenidos en las mismas y dividiéndolos para el número total de cuestionarios aplicados. De esta manera, se obtuvo un puntaje directo que pudo ser ubicado en el cuadro de Puntuación T para calificar la CVL que ofrece el Manual de Aplicación e Interpretación del Instrumento CVT- GOHISALO. En este se establecen los puntajes máximos o mínimos que permiten considerar cuándo una dimensión o sub-dimensión debe ser intervenida.

\section{RESULTADOS}

La recopilación de los puntajes $T$ obtenidos en todas las dimensiones evaluadas, permitieron generar un perfil de los colaboradores del área de ventas en relación a su CVL. Se evidenció que cuatro de las siete dimensiones evaluadas se encuentran sobre el pro- medio (SIT, ST, BLT y DP). Dos dimensiones (IPT y SAT) se ubican dentro de la promedio esperado y solamente una, la (ATL) se encuentra con una puntuación T baja 20 puntos. La administración del tiempo libre (ATL) se ubica por debajo del promedio. (Figura 1).

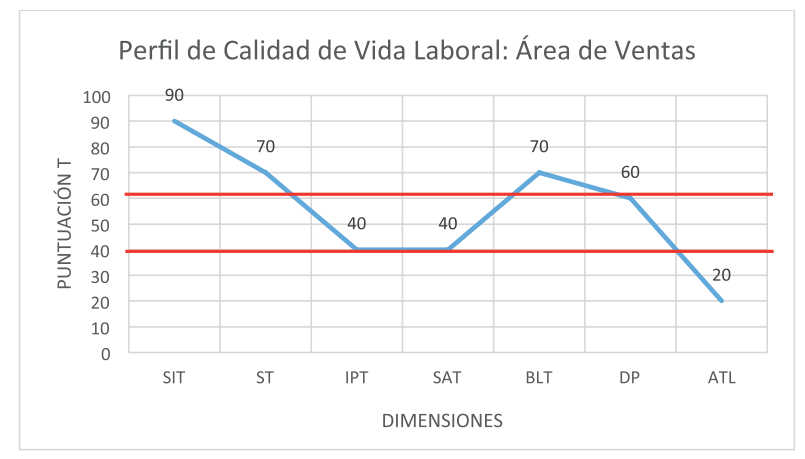

Figura 1. Perfil general de CVL del área de ventas 
Dentro de la dimensión de soporte institucional para el trabajo (SIT) se destaca el resultado obtenido en la pregunta 46 que, con un $76 \%$ de respuestas positivas, ratifican el alto nivel de satisfacción que tienen los colaboradores en cuanto al apoyo que sienten de los superiores para la realización del trabajo. Resultado que destaca el eficaz proceso de comunicación descendente que se presume existe entre superiores y subordinados. Así como el interés que los empleados sienten que tienen sus jefes por ellos y por sus necesidades lo que los mantiene motivados y comprometidos con la organización (Figura 2).

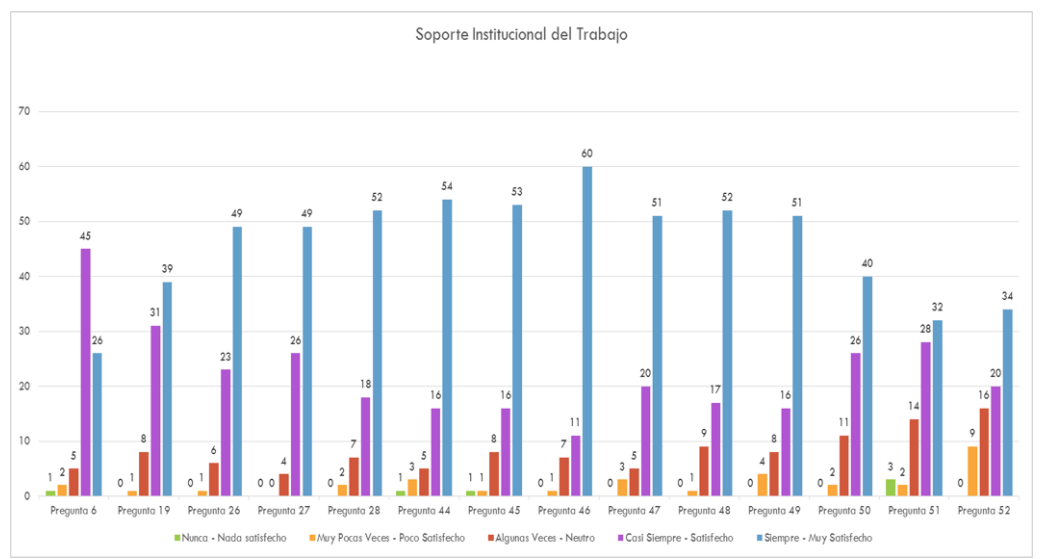

Figura 2. Soporte institucional del trabajo

En lo que se refiere a la evaluación de la dimensión seguridad en el trabajo (ST), se puede mencionar que a pesar de que se obtuvo un puntaje $T$ equivalente a 70 que representa el gran nivel de satisfacción que tienen los colaboradores con respecto a los procedimientos del trabajo, se considera necesario destacar los resultados obtenidos para el indicador de condiciones de higiene y seguridad que abarca la pregunta 11 en donde, 41 respuestas, que representan el $52 \%$ de colaboradores, reflejan la poca satisfacción que estos tienen en cuanto al ruido, iluminación, limpieza, etc. de su lugar de trabajo. Sin embargo, no se debe olvidar que la muestra a la que se tomó los cuestionarios está conformada en su mayoría por visitadores médicos y representantes comerciales cuyo lugar de trabajo en su gran mayoría es su automóvil, siendo la movilización constante un determinante (Figura 3). 


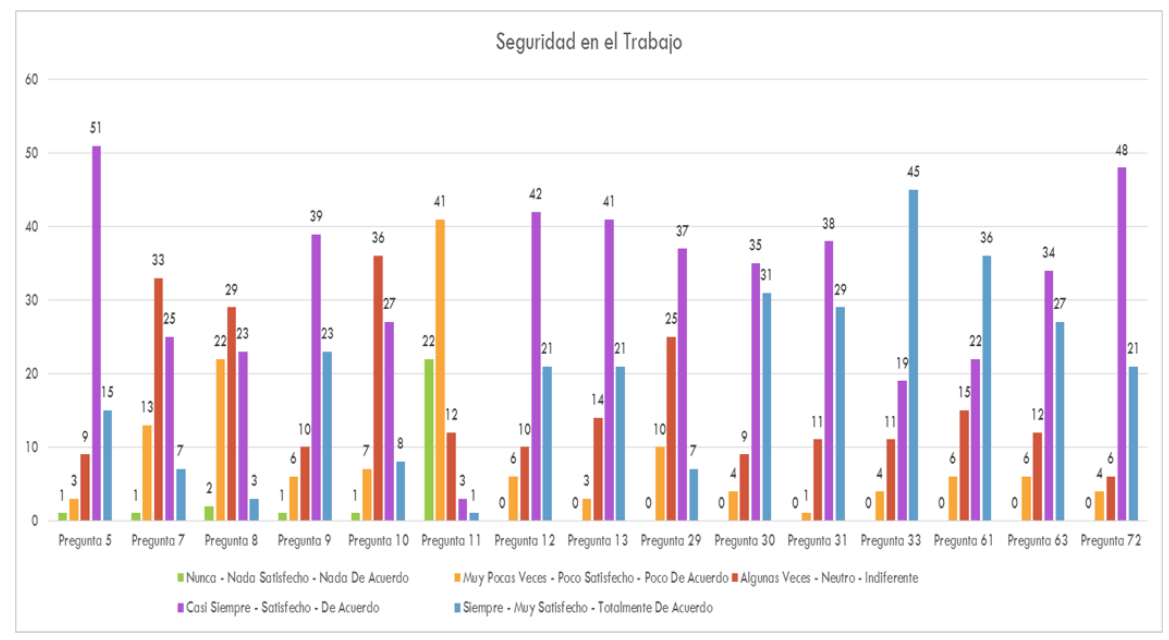

Figura 3. Seguridad en el trabajo

En cuanto a la dimensión de integración al puesto del trabajo (IPT), se obtuvo un puntaje $T$ equivalente a 40 puntos, puntaje considerado bajo según el manual. Identificando a las preguntas 37, 41 y 42 como aquellas con el mayor número de respuestas negativas, las cuales representan falencias en los indicadores de interés por la actividad laboral y apoyo de los compañeros.

Por otra parte, la satisfacción por el trabajo (SAT) fue una de las dimensiones cuyo puntaje $T$ (40) la ubica dentro de la norma; sin embargo, esta presenta una tendencia a la insatisfacción. Identificándose a la pregunta 2 como la única que presenta algún tipo de conflicto, la cual hace referencia a la duración de la jornada que, con 45 respuestas neutras, demuestra que el $57 \%$ de la población se encuentra indiferente en cuanto a la dedicación que le prestan al trabajo.

La dimensión de bienestar logrado a través del trabajo (BLT) es considerada como una de las dimensiones más aceptada por los empleados con una puntuación T de 70 puntos, en donde se destacan los resultados obtenidos en las preguntas 73, 34 y 38 que con un $95 \%$ de respuestas positivas recalcan el auto-compromiso que los colaboradores de esta área tienen con su trabajo e institución, sintiéndose totalmente identificados con los objetivos de la empresa (Figura 4). 


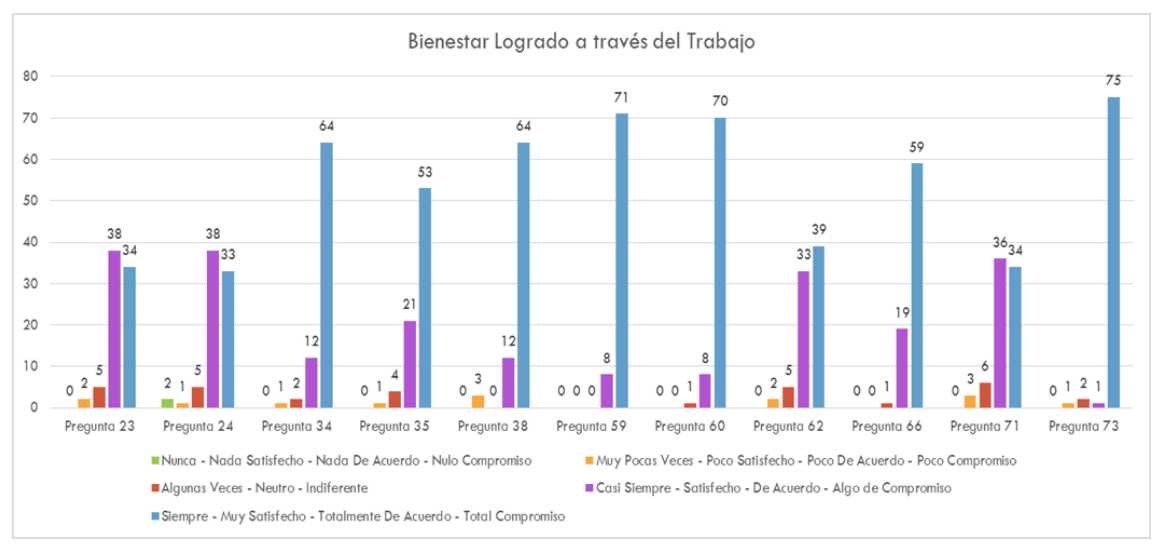

Figura 4. Bienestar logrado a través del trabajo

Articulado a estos resultados se encuentran aquellos obtenidos en la dimensión del desarrollo personal (DPT), que con una puntación T de 60 puntos reflejan el buen ánimo, optimismo y amabilidad de los trabajadores, además de demostrar que ellos están logrando sus metas gracias a que estas son claras, alcanzables y loables. Se evidencia en la pregunta 74 que con un $95 \%$ de empleados que representan a las 75 respuestas alcanzadas, refleja el total compromiso que los colaboradores sienten hacia el logro de los objetivos de la empresa.
Finalmente, la última dimensión evaluada fue la de administración del tiempo libre (ATL), cuyos resultados han permitido conocer que esta es la dimensión que más conflictos presenta para el personal del área de ventas ya que la puntuación T obtenida en la misma es la más baja de todas (20 puntos). Lo que, según el manual, es consecuencia de que los trabajadores no tengan una buena administración del tiempo libre, prolonguen su jornada laboral o suelan llevar trabajo a la casa, lo que repercute en su descanso o recreación (Figura 5). 


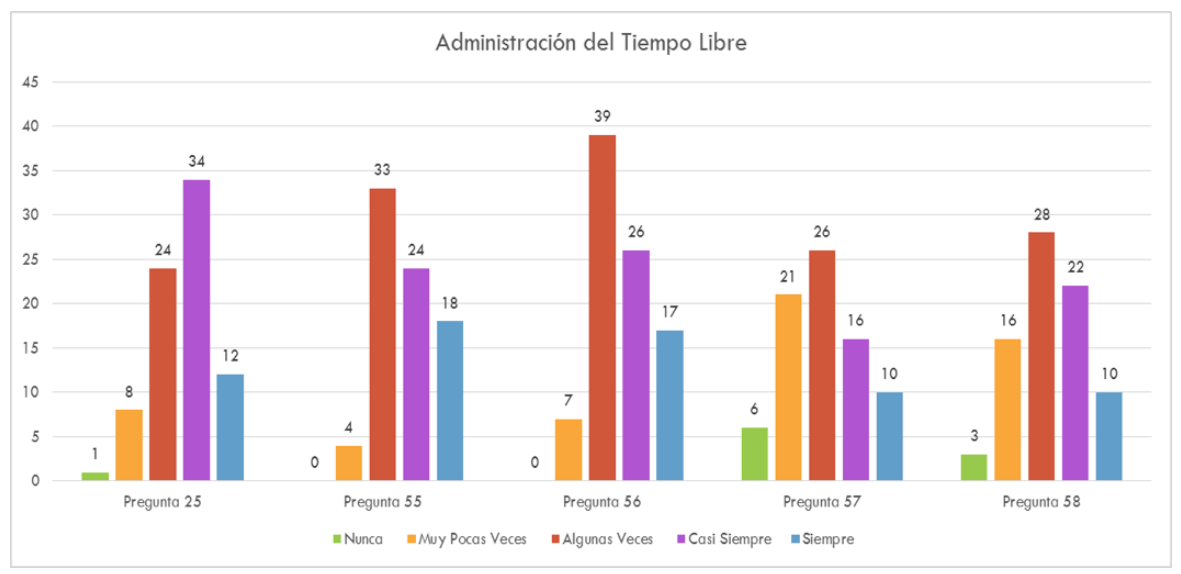

Figura 5. Administración del Tiempo Libre

La Administración del Tiempo Libre está compuesta por dos dimensiones: la planificación del tiempo libre y el equilibrio entre trabajo y familia. En cuanto a la sub-dimensión de planificación del tiempo libre, esta se encuentra compuesta por dos indicadores: la necesidad de trabajar en casa evaluada en la pregunta 25, en donde 34 personas que representan al $43 \%$ de los emplea- dos consideran que casi siempre deben llevar trabajo a casa, factor que influye negativamente en el balance trabajo-familia, y el cumplimiento de actividades programadas que con un $42 \%$ de respuestas demuestran que estas pueden ser llevadas a cabo solamente en algunas ocasiones ya que su trabajo muchas veces se lo impide.

\section{DISCUSIÓN DE RESULTADOS}

Actualmente, nuestra sociedad se interesa cada vez más por el bienestar, la salud y la mejora de la calidad de vida; en esto el trabajo tiene un papel muy importante ya que representa uno de los factores más condicionan- tes para nuestras vidas, relaciones e inclusive nuestra salud. En el año 2009, González, Hidalgo, Salazar y Preciado elaboraron un modelo de calidad de vida en el trabajo que se basa en la teoría de la motivación de Maslow. 
Esta teoría supone que los trabajadores valoran objetiva y subjetivamente el grado en que sus necesidades sociales, de seguridad, autoestima y autorealización se logran por medio del trabajo; planteando además un concepto multidimensional integral de la CVL (González, Hidalgo, Salazar \& Preciado, 2009); (González, Hidalgo, Salazar \& Preciado, 2010); (Patlán, 2016).

El presente estudio determina el perfil de CVL del área de ventas de una farmacéutica en la ciudad de Quito, obtenido a través del instrumento GOHISALO. Se destaca el bajo puntaje obtenido en la administración del tiempo libre. Es importante enfatizar que se trabajó con una población netamente comercial, cuya característica principal es la industria farmacéutica, y en cualquier sector empresarial es no tener un horario de trabajo establecido.

Granados (2011) plantea que las personas con jornadas de trabajo muy prolongadas experimentan dificultades para mantener sus relaciones sociales y familiares normales. La conciliación de actividades diversas en el ámbito trabajo-familia es una base sustancial para el ajuste psicológico del individuo (Jiménez Figueroa, Mendiburro Subiabre, \& Olmedo Fuentes, 2011). Además, este factor supone un impedimento no solo para el esparcimiento y mantenimiento de buenas relaciones familiares y socia- les, sino también para la actualización profesional, a más de los problemas de salud que puede generar.

Actualmente, el trabajo y la familia se constituyen en las dos esferas fundamentales donde actúa el ser humano y a las cuales dedica su mayor parte de tiempo y recursos, por lo que todo lo que sucede en uno de estos dos ámbitos afectará al otro de manera bidireccional (Casas, Repullo, \& Cañas, 2002).

Greenhaus, Collins y Shaw (2003) establecen que para lograr una conciliación entre los roles familiares y laborales se necesita lograr el ajuste y equilibrio en tres componentes: tiempo, niveles de compromiso y lograr un equilibrio en la satisfacción. Es decir, dedicar similar cantidad de tiempo al trabajo y a los papeles familiares, así como al compromiso psicológico con la familia y el trabajo y mantener un equilibrio y satisfacción en ambos dominios.

Como se puede observar es indudable la relación que existe entre la duración de la jornada laboral y la administración del tiempo libre, dimensiones que influyen en la satisfacción de los colaboradores ante su trabajo y que por ende afectan a la calidad de vida laboral de los mismos. Esta relación brinda a la organización pautas para trabajar en medidas que permitan mejorar estos aspectos y cuidar del bienestar de los colaboradores de la organización. 
Como sostiene Peiró y otros, (2015) desde la psicología del trabajo y de las organizaciones se ha brindado aportaciones científicas significativas para mejorar el bienestar de las personas en sus distintos entornos laborales. La Organización Panamericana de la salud, considera que los ambientes laborales, en donde el individuo generalmente pasa la tercera parte de su vida, deben ser promotoras de salud, un bien preciado por los ciudadanos, comunidades y países. En entornos saludables se aumentan la productividad, la motivación, espíritu de equipo, la satisfacción y la calidad de vida en general (Barros Casas \& Paravic, 2006).

\section{CONCLUSIONES}

A partir de los hallazgos realizados en esta investigación y siguiendo el modelo de González, Hidalgo, Salazar y Preciado, se puede concluir que la CVL es un concepto multidimensional que se completa cuando el colaborador, a través de la percepción de su trabajo, cubre sus necesidades personales entre las que se encuentran la satisfacción por su labor y la administración del tiempo libre. Siendo esta última una de las más valoradas por los colaboradores del área de ventas de esta empresa farmacéutica, quienes perciben la falta de conciliación entre el trabajo y la familia como una de las principales causas de insatisfacción en esta investigación. Por esto, la empresa debe considerar la utilización de políticas pro trabajo-familia que tienen una alta incidencia en la satisfacción, tanto como en la disminución de los conflictos ocasionados por ambos roles. Sin embargo, cada individuo constituye un mundo y no todas las políticas, prácticas y programas podrían unificarse, es importante el diálogo con otros campos como el de la psicología, psicología organizacional, sociología, administración, antropología, entre otros para plantear estrategias que cubran necesidades en relación a la crianza de hijos, corresponsabilidad, la función parental, espacios de ocio y recreación.

La duración extensa de la jornada laboral (característica propia de las sociedades pos-modernas) causa insatisfacción a los colaboradores del área de ventas, misma que tiene una estrecha relación con la administración del tiempo libre ya que la extensión de dicha jornada está impidiendo que los empleados cumplan con las actividades planificadas fuera del horario laboral. Este hecho de que colaboradores tengan poco tiempo de convivencia con sus familias, genera un conflicto su- 
mamente grave entre la vida laboral y la vida familiar, lo que puede ocasionar varios problemas a la organización.

Finalmente, cabe señalar conforme Peiró et al. (2015), por aproximadamente 100 años desde la psicología del trabajo y de las organizaciones se ha generado teorías y modelos que buscan mejorar el bienestar de los colaboradores, sin dejar de promover la productividad. Sin embargo, en los últimos años se han puesto en riesgo estas prácticas debido al modelo capitalista predominante, lo cual ha hecho difícil mantener los logros en relación a condiciones de trabajo y calidad de vida laboral, una "sostenibilidad del bienestar laboral". 


\section{BIBLIOGRAFÎA}

Barros Casas, S., \& Paravic, K. T. (2006). Promoción de la salud y un entorno laboral saludable. Revista Latinoamericana Enfermagem 14 (1), 136141.

Casas, J., Repullo, J., \& Cañas, S. (2002). Dimensiones y medición de la calidad de vida laboral en profesionales sanitarios. Revista Administración Sanitaria.

Chiang Vega, M. M., \& Krausse Martínez, K. A. (2009). Estudio empirico de la calidad de vida laboral, cuatro indicadores: satisfacción laboral, condiciones y medioambiente del trabajo, organización e indicador global sector público y privado. Desarrollo, aplicación y validación del instrumento. Horizontes Empresariales, 23-50.

Chiavenato, I. (2007). Administración de recursos humanos: el capital humano de las organizaciones. Mexico D.F: McGraw-Hill.

Figueroa, A. J. (2008). Factores laborales de equilibrio entre trabajo y familia: medios para mejorar la calidad de vida. Universeum, 23 (1), 116-133.

Gestoso, C. (2000). Psicología del trabajo para las relaciones laborales. España: Mc Graw Hill.
Gómez, M. A. (2010). Calidad de vida laboral en empleados temporales del valle de Aburrá - Colombia. Revista Ciencias Estratégicas, 225236.

González Baltazar, R., Hidalgo Santacruz, G., León Cortés, S. G., Contreras Estrada, M. I., Aldrete Rodriguez, M. G., Hidalgo González, B. J., \& Barrera Vega, J. A. (2015). Relación entre género y calidad de vida en profesionales de la salud. Psicogente 18 (33), 52-65.

González, R., Hidalgo, G., Salazar J., \& Preciado, M. (2009). Instrumento para medir la Calidad de Vida en el Trabajo: CVT-GOHISALO. Manual para su aplicación e interpretación. IISO. González, R., Hidalgo, G., Salazar, J., \& Preciado, M. (2010). Elaboración y validez del instrumento para medir calidad de vida en el trabajo "CVT-GOHISALO". Ciencia \& Trabajo, 332-340.

Granados, I. (2011). Calidad de vida laboral: historia, dimensiones y beneficios. Revista IIPSI, 271-276.

Greenhaus, J., Collins, K., \& Shaw, J. (2003). The relation between work-family balance and quality of life. Journal of Vocational Behavior, 510-531.

Guerrero, J., \& Puerto Barios, Y. I. (2007). Productividad, trabajo y salud: la 
perspectiva psicosocial. Revista Colombiana de Psicología, 16, 203234.

Jiménez Figueroa, A., Mendiburro Subiabre, N., \& Olmedo Fuentes, P. (2011). Satisfacción familiar, apoyo familiar y conflicto trabajo-familia en una muestra de trabajadores chilenos. Avances en Psicología Lationameriana Vol. 29(2), 317-329.

Manrique, K. (2013). Desarrollo de Sistemas Socio Técnicos en el área de Seguridad y Salud en el Trabajo de una empresa de servicio. Lima: Pontificia Universidad Católica del Perú.

Martínez Gamarra, M., \& Ros Mar, R. (2010). De la calidad de vida laboral a los riesgos Psicosociales: Evaluaciòn de la calidad de vida laboral. Acciones e investigaciones sociales, 5-55.

Martínez San Esteban , J., \& Calvo Lluch, À. (2014). Calidad de vida percibida y su relaciòn con la práctica de actividad física en el ámbito laboral. Restos. Nuevas tendencias en Educación Física, Deporte y Recreación, 53-57.

McMillan, H., Lane, M., \& Atchley, K. (2011). Constructs of the work/ life intercance: a synthesis of the literatura and introduction of the concept of work/life harmony.
Human Resource Development Review , 6-25.

Moreno, I., Cuevas, M., \& González, J. (2009). Validaciòn y adaptación del cuestionario de calidad de vida en el trabajo (QWLQ) y del cuestionarios genérico de estrés en el trabajo (GJSQ). Revista Interamericana de Psicología Ocupacional, 56-80.

Organización Internacional del Trabajo. (01 de 04 de 2017). OlT.org. Obtenido de OlT.org: http://www.oit. org/global/topics/decent-work/ lang--es/index.htm

Ortega Farias, A., Rodríguez Soto, D., \& Jiménez Figuero, A. (2013). Equilibrio trabajo-familia: corresponsanilidad familiar y autoeficacia parental en trabajodres de una empresa chilena. Diversitas Vol. 9 Issue 1, 55-64.

Pasamar, S., \&Valle, R. (2011). Conciliación de la vida profesional personal en empresas españolar. Mito o realidad? Universia Business Review Primer trimestres, 14-31.

Patlán, J. (2016). Calidad de vida en el trabajo. Ciudad de México: Editorial El Manual Moderno S.A. de C.V.

Peiró, J. M. (1996). Tratado de psicología del trabajo. Síntesis.

Peiró, J., Tordera, N., Lorente, L., Rodriguez, I., Ayala, Y., \& Latorre, F. (2015). Bienestar sostenible en el 
trabajo: conceptualización, antecedentes y retos. Psiencia:Revista Latinoamericana de Ciencias Psicológicas, 133-135.

Salgado, M. E. (2011). Balance vida-trabajo de las mujeres gerentes que trabajan en empresas privadas del área metropolitana de Caracas. Caracas: Universidad Católica Andrés Bello .

Secretaria Nacional de Planificación y Desarrollo. (01 de 04 de 2017). Senplades. Obtenido de Senplades: http://www.buenvivir.gob.ec/
Segurado, A., \& Agulló, E. (2002). Calidad de vida laboral: hacia un enfoque integrador desde la psicología social. Psicothema, 828-836.

Torres Martos, M. J., \& Onsalo, R. (2012). Impacto de la ley de igualdad en el contenido de la negociacion colectiva del sector andaluz de la construcción. Intangible Capital, 8 (2), 447-471.

Valadez, L. (2012). Balance trabajo-familia: México y Nuevo León en perspectiva internacional. Instituto Municipal de la Familia de San Pedro Garza García, 50. 